\title{
Genomic Imprinting: Antagonistic Mechanisms in the Germ Line and Early Embryo
}

\author{
A.M. FEDORIw, N.I. EnGEL, AND M.S. BARTOLOMEI \\ Howard Hughes Medical Institute and Department of Cell and Developmental Biology, \\ University of Pennsylvania School of Medicine, Philadelphia, Pennsylvania 19104
}

\begin{abstract}
Proper mammalian development requires the contributions of both a maternal and paternal genome (Barton et al. 1984; McGrath and Solter 1984). This unique constraint is due to a subset of genes whose monoallelic expression is dependent upon the parent of origin. Therefore, despite identical genetic information, alleles of such imprinted genes can be discriminated from one another by transcriptional activators or repressors in the nuclei of all somatic cells. Also implied by this phenomenon are mechanisms in each gamete that must establish a transcriptional state for an allele, one that is capable of being interpreted and stably maintained throughout the lifetime of the organism. Correct establishment and maintenance of these states is paramount, as misregulation of imprinted genes is associated with many developmental defects as well as with numerous cancers (Lalande 1996; Tycko 1999). Recent data have uncovered cis-elements and trans-factors that control the regulation of various imprinted genes in the soma. However, the mechanisms that govern establishment of imprints during gametogenesis remain incompletely understood.
\end{abstract}

The organization of imprinted genes within the genome provides several clues about their regulation. Approximately 70 imprinted genes have been identified in mammals (http://www.mgu.har.mrc.ac.uk/research/imprinted/). Not only is their imprinted expression well conserved between mouse and man, but their genomic organization is as well, as imprinted genes are often associated in clusters throughout the genome (Verona et al. 2003). The high level of conservation between imprinted expression patterns and clustered organization suggests a functional link between the two. Chromosomal rearrangements or deletions within these clusters often cause misregulation of linked genes, providing further evidence of a functional connection between their organization and regulation (Reik and Maher 1997). Central to their allele-specific expression, differential DNA methylation has been associated with almost all imprinted loci (Brannan and Bartolomei 1999). Targeted mutagenesis has shown that these differentially methylated domains (DMDs; also differentially methylated regions, DMRs) are critical to the regulation of neighboring imprinted genes (Thorvaldsen et al. 1998; Fitzpatrick et al. 2002; Lin et al. 2003). Because it is stable and heritable through cell division, DNA methylation is the leading candidate for the imprinting "mark," the epigenetic coding that distinguishes one allele from another.
The critical importance of DNA methylation to imprinted gene regulation has been demonstrated in mice that carry mutations in several members of the DNA methyltransferase (Dnmt) family, the enzymes responsible for methylating DNA in mammals. Hypomorphic mutations of the first such enzyme identified, Dnmt1, have demonstrated that this protein is primarily involved in maintaining methylation at both imprinted and nonimprinted loci (Li et al. 1993). An oocyte-specific, maternally loaded alternative splice form of Dnmt1, Dnmt1o, functions to maintain methylation during a brief window of preimplantation development (Howell et al. 2001).

The identification and characterization of three Dnmt3 genes has linked these related proteins to establishment of imprints, as these genes are highly expressed during gametogenesis in both parents (La Salle et al. 2004). Compound mutations of Dnmt3a and Dnmt3b genes have uncovered a vital, yet possibly redundant, role in establishing methylation imprints (Okano et al. 1999). More recently, conditional null alleles of Dnmt3a have demonstrated an essential role for this de novo methyltransferase in the acquisition of many, but not all, methylation imprints in both parental germ lines (Kaneda et al. 2004). Alternative forms of Dnmt3a have also been identified, potentially adding greater complexity to the establishment of methylation imprints (Chen et al. 2002). Interestingly, the only member of this family that does not have DNA methyltransferase activity in vitro, Dnmt3l, is required for establishment of methylation at imprinted loci at least in oocytes (Bourc'his et al. 2001). Biochemical data suggest that Dnmt31 may exert its activity by interacting with Dnmt3a and 3b (Hata et al. 2002). Therefore, Dnmt31 may provide sequence specificity to the other Dnmt3 proteins.

Differentially methylated domains can be divided into those that are maternally or paternally hypermethylated. The majority of known DMDs, such as those for the Snurf-snrpn, Igf2r, Kcnq1ot1, and Peg3 loci, are maternally hypermethylated, while the differentially methylated regions of $H 19$ and Rasgrfl are paternally hypermethylated (Stöger et al. 1993; Plass et al. 1996; Shemer et al. 1997; Tremblay et al. 1997; Li et al. 2000; Fitzpatrick et al. 2002). Recent work has focused on the paternally hypermethylated H19 DMD as a paradigm not only for the regulation of imprinting, but for its establishment as well.

H19 and Insulin-like growth factor 2 (Igf2) are two closely linked, yet oppositely imprinted genes (Bar- 


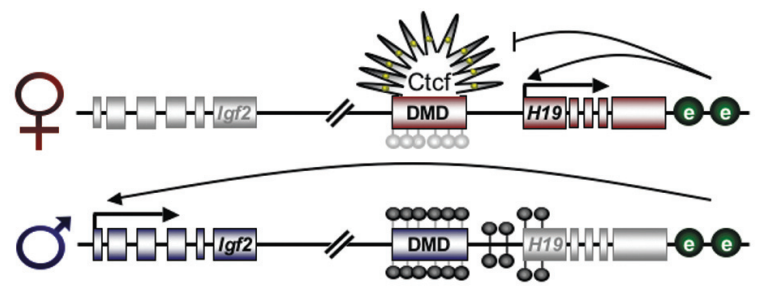

Figure 1. Imprinted expression of the H19/Igf2 locus. Methylation of the DMD regulates the expression of $H 19$ and Igf 2 . The DMD is maternally hypomethylated (open circles) and paternally hypermethylated (filled circles). On the maternal allele, the DMD acts as an enhancer-blocking element through the binding of Ctcf. The shared enhancers (e) can only activate the $H 19$ promoter. On the paternal allele, methylation of the DMD prevents Ctcf binding, and Igf 2 is expressed. The methylation in the DMD spreads to the H19 promoter during development, thereby preventing its expression.

tolomei et al. 1991; DeChiara et al. 1991). H19 is located approximately $100 \mathrm{~kb}$ downstream of the $\operatorname{Igf} 2$ promoter; the DMD lies $2 \mathrm{~kb}$ upstream of the $H 19$ transcriptional start site (Fig. 1). Analyses from several laboratories have demonstrated that the DMD is required on both parental chromosomes for proper imprinted expression. Various targeted deletions and mutations have shown that in somatic tissues, the DMD acts as a switch that directs transcriptional activity by regulating access of enhancers that are shared by both genes (Thorvaldsen et al. 1998; Srivastava et al. 2000). On the paternal chromosome, where the DMD is hypermethylated, $H 19$ is silent and Igf 2 is expressed. The repression of $\mathrm{H} 19$ is thought to occur through the recruitment of repressive factors by the hypermethylated DMD. On the maternal allele, hypomethylation of the DMD results in the formation of an enhancer-blocking element that limits the action of the enhancers to the $H 19$ promoter, thereby preventing activation of $\operatorname{Ig} 2$. The function of the DMD on the maternal allele is mediated by the enhancer-blocking protein, Ctcf (Bell and Felsenfeld 2000; Hark et al. 2000; Kanduri et al. 2000; Szabo et al. 2000; Schoenherr et al. 2003). Recent work from our laboratory has shown that the requirement for Ctcf on the maternal allele extends to a critical period in oocyte growth when maternally hypermethylated DMDs acquire their characteristic levels of methylation (Fedoriw et al. 2004).

In this review, we summarize what is known about the establishment of DNA methylation on DMDs during gametogenesis. We also discuss mechanisms that are hypothesized to initiate and maintain differential methylation at imprinted loci.

\section{METHYLATION OF DMRS IN THE GAMETES}

One of the main implications of the existence of imprinted genes is that mechanisms must exist within each germ line to establish the future parental identity of a given locus. Presently, we know little about what molecules are involved in these processes and how the signature patterns are generated. Even the full nature of the marks remains elusive. As one of the first epigenetic modifications identified, DNA methylation has been most extensively described for several loci in the germ lines. Recently, the expression patterns of the Dnmt family of proteins has been shown to be quite different in the male and female germ line (La Salle et al. 2004). These data, along with the divergent processes that produce male and females gametes, provide ample opportunity for differential epigenetic modification. What remains to be shown, however, is what signals and factors cooperate to methylate a DMR exclusively in a single parental germ line.

Before a parental imprint can be conferred upon a given locus, any existing epigenetic modifications must be removed. The demethylation of various DMRs has been extensively studied in the developing mouse embryo. The allocation of the germ line occurs early in development; after their specification around 7 days postcoitum (dpc), primordial germ cells (PGCs) migrate to and populate the primitive gonad by $10.5 \mathrm{dpc}$ (McLaren 2003). Erasure of DNA methylation at imprinted loci is complete before $12.5 \mathrm{dpc}$ (Hajkova et al. 2002; Lee et al. 2002), coincident with the time when the PGC genome is globally undermethylated compared with the somatic genome (Monk et al. 1987). The paternal $H 19$ allele is hypomethylated by $12.5 \mathrm{dpc}$ in both germ lines (Davis et al. 2000), an observation that may point to a common mechanism of erasure. Completion of erasure also coincides with cessation of PGC proliferation. Thus, methylation imprints are erased before further differentiation and proliferation of the male and female gametes.

At $12.5 \mathrm{dpc}$, gametogenesis becomes sex-specific and the processes that occur in the developing gonads differ greatly. In the developing male germ line, hypermethylation of select DMRs as well as intracisternal A particle (IAP) repetitive elements occurs by late gestation (Brandeis et al. 1993; Walsh et al. 1998; Davis et al. 2000). Concurrent with its proposed role in directing de novo methylation of imprinted regions (Hata et al. 2002), Dnmt3l expression peaks during the establishment of DNA methylation imprints before birth in the male germ line. Among DMRs that are paternally methylated, the acquisition of methylation has been best studied at the H19 DMD (Davis et al. 1999, 2000; Ueda et al. 2000). The paternal allele is almost fully methylated by $13.5 \mathrm{dpc}$, shortly after demethylation is complete. Unexpectedly, significant levels of methylation are observed on the maternal DMD only after $16.5 \mathrm{dpc}$ (Davis et al. 2000). This observation implies that although both alleles are hypomethylated, they continue to retain some parental identity. Additionally, this difference is sufficient to be recognized by Dnmts, or other factors, in the developing gamete. The nature of this difference is unknown, yet it may reflect differences in chromatin structure (such as bound Ctcf, histone modifications, etc.) that make the paternal allele more susceptible to methylation than the maternal allele (Davis et al. 2000; Ueda et al. 2000).

In contrast to the male germ line, oogonia proliferate and become meiotically arrested before birth. These incompetent, or nongrowing (ng), oocytes are globally undermethylated compared to the similar embryonic stage in the male. Additionally, DMRs are hypomethylated at this point (Lucifero et al. 2002). At birth, oocytes begin to 
grow and differentiate and gain competence to undergo the first meiotic division. During oocyte growth, and coincident with an increase in Dnmt31 mRNA levels, the DMRs of various maternally hypermethylated loci acquire their methylation (Lucifero et al. 2004).

The timing of DNA methylation at imprinted loci during oocyte growth has been characterized by several groups (Lucifero et al. 2002, 2004; Obata and Kono 2002). Their data show that not all DMRs acquire methylation simultaneously. The Pegl DMR, for example, does not become completely hypermethylated until the oocyte is nearly fully grown. Others, like the Peg3 DMR and $\operatorname{Igf} 2 r$ DMR2, acquire methylation much earlier. Interestingly, de novo methylation of Snurf-snrpn DMR1 in the female germ line parallels that observed for the H19 DMD in the male germ line with the maternal allele being hypermethylated earlier than the paternal allele (Lucifero et al. 2004). De novo methylation of maternally methylated DMRs is complete by the time the oocyte has fully matured.

\section{MODELS FOR IMPRINT ESTABLISHMENT}

Along with studies determining the timing of DNA methylation, cis-elements required to maintain imprints in the soma have also been well characterized. What remains to be elucidated are the signals required for establishment of a maternal or paternal identity in the germ line. Are the states actively determined in both germ lines or does one represent a default state for a particular locus? Two general models can be postulated to explain how these states are established. One possibility is the presence of sex-specific germ line factors that could target the de novo DNA methylation machinery to those DMRs that are to be hypermethylated (Fig. 2A). The other germ line would not express the same factor and, therefore, the DMR would remain unmethylated in that gamete. Alternate splice forms or differential expression of accessory factors would be sufficient to provide sequence specificity for Dnmt proteins in one germ line, but not the other. For example, the $\operatorname{Ig} f 2 r$ DMR2 would remain hypomethylated during spermatogenesis because the protein(s) that direct its de novo methylation would be ex-
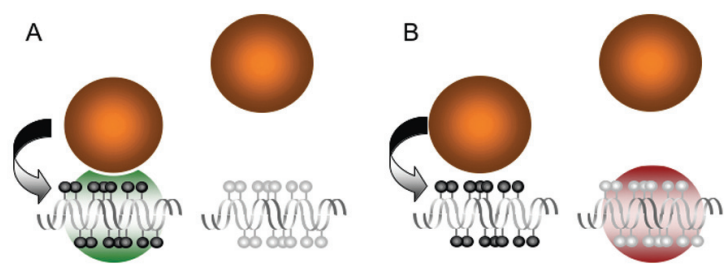

Figure 2. Models for establishment of methylation imprints. Two models for the differential methylation of imprinting control regions in the germ lines. (A) Sequence-specific factors (green circle) are expressed in only one germ line. These factors direct activity of de novo Dnmts (orange circles). Because the targeting factors are not expressed in the other germ line, the target sequence is not methylated. (B) Conversely, Dnmts recognize the CpG-rich sequences in both germ lines but protective factors (red circles) are only expressed in one developing gamete. pressed only during oocyte growth. An alternative model posits that both germ lines could have similar potential for directing methylation to imprinted regions. However, sex-specific expression of factors that protect or exclude a locus from de novo methylation would be the primary determinant of a hypomethylated or hypermethylated DMR (Fig. 2B). Because this protective effect would be conferred in only one germ line, a differential state of methylation would be established.

\section{ESTABLISHING THE MATERNAL IDENTITY OF THE H19 DMD}

Despite the large number of targeted mutations that have revealed essential cis-regulatory elements at the H19 locus, none has identified an element that is required for establishment of the methylation pattern during gametogenesis. In contrast, deletion of an element adjacent to the Rasgrf1 DMD results in a maternal-like hypomethylated state in sperm (Yoon et al. 2002). Therefore, it is unclear whether the H19 locus harbors such an element. Alternatively, the current array of mutations may have abolished a signal that is required for both preventing and attracting methylation.

Several laboratories have proposed models for how the imprint is established at the H19 DMD (Pfeifer 2000; Thorvaldsen and Bartolomei 2000). One possibility is that factors in the male germ line direct methylation to the H19 DMD exclusively during spermatogenesis, overriding the default hypomethylated state. Here, the H19 DMD would remain unmethylated during oocyte growth because of the absence of targeting factors. Furthermore, Ctcf would only be required on the maternal allele after fertilization to establish an insulator. Another model hypothesizes that Ctcf would be required to protect this locus from de novo methylation during oogenesis. In this case, Ctcf would be bound to the DMD during oocyte growth, establishing the enhancer-blocking chromatin structure prior to fertilization.

The latter model is particularly attractive because a protective role for a transcription factor has been described previously. The ubiquitous transcriptional activator, $\mathrm{Sp} 1$, has been shown to prevent de novo methylation of its binding site in a transgene model (Brandeis et al. 1994; Macleod et al. 1994). Could Ctcf play a similar role? Since Ctcf is expressed when methylation imprints are being established in the female germ line, we postulated that Ctcf is required for the H19 DMD to remain hypomethylated during this period. If so, then oocytes that have been depleted of Ctcf protein should bear a hypermethylated H19 DMD.

We used a transgenic-RNA-interference (RNAi)-based approach to target Ctcf mRNA for degradation specifically in growing oocytes (Fedoriw et al. 2004). We developed a series of transgenic lines that depleted Ctcf mRNA and protein to varying levels. This system provided us with a means to assess how vulnerable the H19 DMD is during a particularly active time of methylation acquisition at other imprinted loci. In fact, oocytes from transgenic females with the greatest reduction of Ctcf did acquire significant methylation at the DMD, rivaling the 
level seen on the wild-type paternal allele. This observation suggested that Ctcf is the critical factor in determining the maternal hypomethylated identity.

Since depletion of a broadly required transcription factor or essential chromatin component could lead to nonspecific responses, we also analyzed these oocytes for other potential defects in methylation. We have not observed abnormal methylation of other loci, nor has depletion of Ctcf led to loss of methylation at maternally hypermethylated DMRs (Fedoriw et al. 2004). We have also found that the H19 DMD does not become methylated simply in the presence of other double-stranded RNAs (dsRNAs); in oocytes from a transgenic female carrying a similar transgene specific for the Mos mRNA (Stein et al. 2003), the H19 DMD remained hypomethylated (A. Fedoriw, unpubl.). These data show that the hypermethylation observed at the H19 DMD is not due to nonspecific effects; rather, the hypermethylation is the consequence of the DMD losing the protection it requires to repel the activity of Dnmts.

Recently, Loukinov and coworkers identified a Ctcflike protein designated Boris (Brother of the Regulator of Imprinted Sites) (Loukinov et al. 2002). Boris is expressed exclusively in type A spermatocytes, which are reported to lack Ctcf. Additionally, these cells appear deficient for methylated DNA, as assayed by anti-5-methylcytosine antibody staining, although these data conflict with those reported by several other groups (Davis et al. 2000; Ueda et al. 2000). Loukinov and coworkers have hypothesized that Boris is responsible for the hypermethylated state of the DMD in the male germ line by either sequestering Ctcf or displacing it from its binding sites and allowing Dnmts to methylate those sites. In the models outlined in Figure 2, Boris may be an example of a targeting factor. We observed no ectopic expression of either Boris protein or mRNA in oocytes from transgenic females, suggesting that the methylation we observed in our system was unlikely to be mediated through this molecule (A. Fedoriw, unpubl.).

Our results showing ectopic $H 19$ methylation were rather surprising considering that targeted mutations of the DMD that ablate binding of Ctcf, as assayed by electrophoretic mobility shift experiments, resulted in methylation of the DMD only later during embryonic development (Pant et al. 2003; Schoenherr et al. 2003; Szabo et al. 2004). The DMD remained hypomethylated in fully grown oocytes and hypermethylated in sperm. Several possible explanations could account for this discrepancy. First, the four Ctcf binding sites that mediate the protection of the DMD in the soma may not exhibit the same function in the germ line. Other unidentified Ctcf binding sites located within or perhaps distant from the DMD may be responsible for conferring this function. Ctcf can bind a wide range of sequences, presumably through the use of distinct zinc fingers (Filippova et al. 1996). Therefore, the exact nucleotide sequences that Ctcf contacts in oocytes may be distinct from those required for the enhancer-blocking function in the soma. Second, it is possible that depletion of Ctcf results in misregulation of genes such as Dnmts, whose abnormal expression may lead to the observed phenotype. Although we are currently ex- ploring this possibility, we do not think that it is a likely cause of ectopic methylation. The levels of transcripts for the Dnmt family are higher in the growing oocyte than at any other point during gametogenesis. Therefore, if increased RNA and protein caused nonspecific DNA methylation, it would likely have been detected at other loci, such as the skeletal $\alpha$-actin promoter, which we did not observe (Fedoriw et al. 2004).

Finally, although the H19 DMD acquires a high level of methylation in the Ctcf-depleted oocytes, many DNA strands remain hypomethylated. This may be a consequence of residual Ctcf that is present as the oocytes mature, leaving some protein that can protect the locus. However, as in the early male germ line, the hypomethylated paternal and maternal H19 DMD alleles may be differentially regulated in the growing oocyte. We are currently investigating whether there is an allelic bias to the acquisition of methylation at the H19 DMD in Ctcfdepleted oocytes. Perhaps only one of the alleles is susceptible to methylation at a given point during oogenesis, while the other has a chromatin conformation that is resistant. In this case, the maternally inherited DMD is protected by Ctcf, while the demethylated paternal allele is protected by other means. Therefore, mutant DMD alleles that cannot bind Ctcf would resemble the paternal alleles in the oocyte, and may have another mechanism whereby they remain protected from, or not targeted for, de novo methylation. The observed requirement for Ctcf would not occur in this case until the insulator is established during development. Together with previous observations from the paternal germ line, each allele of $H 19$ or any other imprinted gene may have a unique method for establishing its new parental identity.

\section{THE PATERNAL H19 DMD: ANTAGONISTIC FUNCTIONS WITHIN OVERLAPPING ELEMENTS}

Although the maternal, hypomethylated state of the DMD is actively determined during oocyte growth, hypermethylation of the DMD in the paternal germ line is not necessarily a default state. Rather, the paternal identity of this locus may require specific factors or chromatin modifications to ensure that methylation is properly established, strictly maintained during each cell division, and correctly interpreted in the nucleus of every cell.

Data from our lab have demonstrated that the enhancer-blocking function of the DMD and the hypermethylated, silencing function of the DMD cannot coexist on the same allele (Engel et al. 2004). We generated a targeted allele of the DMD, $H 19^{D M D-9 C G}$, in which we mutated $\mathrm{CpG}$ dinucleotides in the Ctcf-binding sites. This allele was capable of binding Ctcf even in the presence of methylation (Fig. 3). When inherited paternally, these mutations did not interfere with the acquisition of methylation at this locus, as the mutant DMD is hypermethylated in sperm. However, and in contrast to the wild-type paternal allele where the hypermethylated state is strictly maintained, many paternal $H 19^{D M D-9 C G}$ chromosomes become gradually demethylated during development. The apparent instability of the methylation is suggestive 


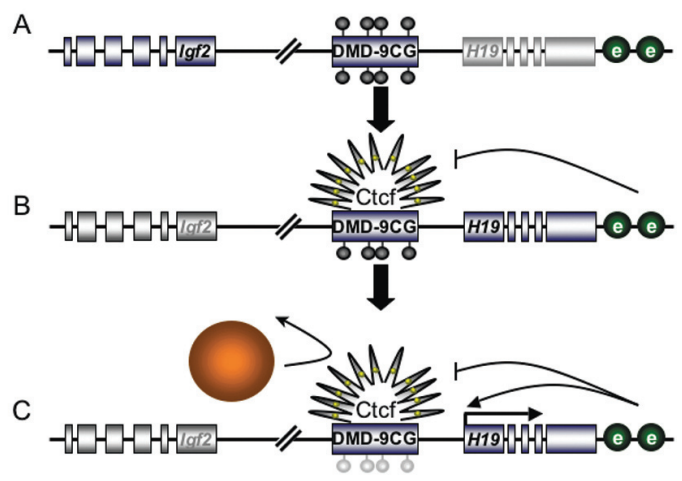

Figure 3. The paternal $H 19^{\text {DMD-9CG }}$ allele activates $H 19$. (A) In sperm, methylation of the mutant DMD is not affected. $(B)$ However, since the mutant DMD can bind Ctcf even when methylated, the mutant DMD will still function as an enhancer blocker. (C) As a consequence, loss of methylation can be observed both at the DMD and at the promoter region. The instability of the hypermethylated state can occur through exclusion of maintenance methylation by Dnmt1 (orange circle).

of an antagonistic relationship between two mutually exclusive functions encoded within the same sequence. The targeted mutations result in an allele that cannot faithfully propagate the paternal epigenotype because it has acquired a property normally restricted to the maternal DMD, i.e., binding of Ctcf, a conclusion supported by studies in transfected embryonic stem cells (Rand et al. 2004). This is the only example of a DMD mutation that specifically affects the paternal allele.

It is quite surprising that mutation of only nine $\mathrm{CpG}$ dinucleotides results in a dramatic reduction in the ability of the paternal allele to retain its methylation state. One possible explanation is that loss of methylation occurs because of a decrease in $\mathrm{CpG}$ density. An alternative hypothesis is that the loss of methylation is dependent upon ectopic Ctcf binding. Ctcf may protect or exclude maintenance DNA methylation by Dnmt1 during embryonic divisions (Fig. 3).

While these two explanations may hold, the loss of methylation on the mutant allele may also reflect an incomplete establishment of the paternal state of the DMD. Perhaps the same sites that function in the maternal germ line as Ctcf-binding sites serve in the paternal germ line as docking sites for other factors. These factors would translate the hypermethylated state of the DMD into associated histone modifications and a resulting chromatin structure would lock in the methylation at this locus. Such an "epigenetic superstructure" could explain the resistance of the H19 DMD to the genome-wide, active demethylation that occurs in the male pronucleus shortly after fertilization and the passive demethylation that occurs thereafter (Rougier et al. 1998; Mayer et al. 2000; Oswald et al. 2000; Santos et al. 2002). Therefore, loss of binding sites (or decrease in $\mathrm{CpG}$ density) would not affect acquisition of methylation at the DMD during spermatogenesis. It would, however, fail to propagate this state during embryogenesis and result in the gradual loss of methylation that we observe. As Boris seems the most likely candidate for such a role in spermatocytes, it will be of great interest to ascertain whether male mice that lack Boris faithfully establish a hypermethylated DMD that can be maintained in the offspring.

\section{CONCLUSIONS}

Imprinted genes are expressed in a parent-of-originspecific manner. DNA methylation appears to be an epigenetic mark that allows two genetically identical alleles to be discriminated. Recently, research has demonstrated how DMDs control their associated imprinted genes. However, the mechanisms that establish a maternal or paternal identity of these DMDs in the germ lines remain poorly understood.

Our results argue that, at least for $H 19$, the hypomethylated state of the maternal DMD is established by Ctcf binding during oogenesis. Thus, Ctcf appears to be a critical factor that distinguishes the H19 DMD from its hypermethylated counterparts in the growing oocyte. It is not yet clear whether a similar mechanism can be extended to other loci. While it has been proposed that Ctcf may be a global regulator of imprint establishment, a role for Ctcf at other imprinted loci remains undefined. Other DMRs that contain Ctcf-binding sites, such as the KvDMR1, are maternally hypermethylated (Kanduri et al. 2002), in contrast to the maternally hypomethylated H19 DMD. This observation suggests that either Ctcf is not itself sufficient to confer a maternally hypomethylated state or the mechanisms required for establishment of a methylation imprint may be unique to each DMR.

Just as a protective mechanism may not function at every imprinted locus, our results do not preclude the possibility that the hypermethylated state is also actively determined. In the $H 19$ paradigm, factors such as Boris may be required to generate a complete paternal imprint. This hypothesis is supported by the inability of the paternally inherited $H 19^{D M D-9 C G}$ allele to maintain a hypermethylated state. Certainly, the complex bipartite function of the Snurf-snrpn DMR2 argues that this locus may also be controlled through active modification in both germ lines (Nicholls and Knepper 2001). Chromosomal abnormalities, most frequently deletions, in the promoter region of the human $S N U R F-S N R P N$ promoter are associated with unique disease phenotypes, depending on the parent from which these lesions are inherited (Nicholls et al. 1999; Cassidy et al. 2000). Collectively, genetic data from these patients have refined the regions responsible for each phenotype, identifying critical domains for imprinting of this locus. The shortest regions of overlap of the different deletions for each syndrome do not themselves overlap, indicative of independent mechanisms functioning to establish the maternal and paternal identities.

Conversely, the imprint at other loci seems to be determined in only one germ line, while the other represents a default state. At such loci, deletion of the DMR would result in an allele reflecting the default state for the locus. This appears to be the case for the Rasgrf1 and Kcnq1ot1 DMRs. The Rasgrf1 DMD is hypermethylated in sperm; however, deletion of adjacent repetitive sequences results in paternal hypomethylation of this domain (Yoon et al. 
2002). Maternal inheritance of this deletion has no effect on Rasgrfl repression. At the Kcnqlot1 locus, the transcription of an antisense RNA (which is repressed by hypermethylation of the maternal KvDMR1) results in long-range repression on the paternal chromosome (Fitzpatrick et al. 2002). Paternal inheritance of a KvDMR1 deletion results in a maternal-like expression pattern of the associated imprinted genes. Maternal inheritance of this mutation has no effect on gene expression. Similarly, only maternal transmission of the IG-DMR deletion at the $D l k 1 / G t l 2$ locus results in a loss of imprinted expression on that chromosome (Lin et al. 2003).

Together, the above observations suggest that all imprints may not be created equal. While active mechanisms may be involved in the establishment of one-or both-imprinted states, it seems that each DMR may be governed by its own rules. Perhaps this diversity reflects the variety of mechanisms DMRs employ to exert longrange transcriptional activation or silencing: Boundaries, enhancer blockers, antisense-RNA, and functional noncoding RNAs have all been connected to imprinted loci. It would be expected that loss of methylation of these control regions would have unique consequences depending on the mode of regulation used by the DMR. At the H19/Igf2 locus, the DMD serves as either an enhancer blocker or silencer, depending on its methylation state. Therefore, each gamete may have evolved a specific mechanism to generate the intended transcriptional state. Imprinted loci controlled by antisense RNA, such as $\operatorname{Ig} f 2 r$, may require only one establishing mechanism since antisense transcription is believed to be the key factor in repressing associated genes. While it remains to be seen whether the models and examples presented can be applied to other imprinted genes, the data described above should provide a better framework for dissecting mechanisms involved in establishing imprinted states.

\section{ACKNOWLEDGMENTS}

We are grateful for the helpful discussions and critical reading of the manuscript by the members of the laboratory. We are also indebted to our many collaborators, particularly Drs. Gary Felsenfeld, Adam West, and Richard Schultz, who contributed to the ideas expressed in this manuscript. This work was supported by NIH grants HD42026 and GM-51279 and the Howard Hughes Medical Institute. A.M.F. was supported by an NIH predoctoral training grant (HD-07516) and N.I.E. is the recipient of a National Research Service Award (HD-41345).

\section{REFERENCES}

Bartolomei M.S., Zemel S., and Tilghman S.M. 1991. Parental imprinting of the mouse $\mathrm{H} 19$ gene. Nature 351: 153.

Barton S.C., Surani M.A.H., and Norris M.L. 1984. Role of paternal and maternal genomes in mouse development. Nature 311: 374 .

Bell A.C. and Felsenfeld G. 2000. Methylation of a CTCF-dependent boundary controls imprinted expression of the Igf2 gene. Nature 405: 482

Bourc'his D., Xu G.L., Lin C.S., Bollman B., and Bestor T.H. 2001. Dnmt3L and the establishment of maternal genomic imprints. Science 294: 2536.
Brandeis M., Kafri T., Ariel M., Chaillet J.R., McCarrey J., Razin A., and Cedar H. 1993. The ontogeny of allele-specific methylation associated with imprinted genes in the mouse. EMBO J. 12: 3669.

Brandeis M., Frank D., Keshet I., Siegfried Z., Mendelsohn M., Nemes A., Temper V., Razin A., and Cedar H. 1994. Sp1 elements protect a $\mathrm{CpG}$ island from de novo methylation. $\mathrm{Na}$ ture 371: 435.

Brannan C.I. and Bartolomei M.S. 1999. Mechanisms of genomic imprinting. Curr. Opin. Genet. Dev. 9: 164

Cassidy S.B., Dykens E., and Williams C.A. 2000. Prader-Willi and Angelman syndromes: Sister imprinted disorders. Am. J. Med. Genet. 97: 136.

Chen T., Ueda Y., Xie S., and Li E. 2002. A novel Dnmt3a isoform produced from an alternative promoter localizes to euchromatin and its expression correlates with active de novo methylation. J. Biol. Chem. 277: 38746.

Davis T.L., Yang G.J., McCarrey J.R., and Bartolomei M.S. 2000. The H19 methylation imprint is erased and reestablished differentially on the parental alleles during male germ cell development. Hum. Mol. Genet. 9: 2885.

Davis T.L., Trasler J.M., Moss S.B., Yang G.J., and Bartolomei M.S. 1999. Acquisition of the H19 methylation imprint occurs differentially on the parental alleles during spermatogenesis. Genomics 58: 18.

DeChiara T.M., Robertson E.J., and Efstratiadis A. 1991. Parental imprinting of the mouse insulin-like growth factor II gene. Cell 64: 849.

Engel N.I., West A.E., Felsenfeld G., and Bartolomei M.S. 2004. Antagonism between DNA hypermethylation and enhancerblocking activity at the $H 19 \mathrm{DMD}$ is uncovered by $\mathrm{CpG}$ mutations. Nat. Genet. 36: 883.

Fedoriw A.M., Stein P., Svoboda P., Schultz R.M., and Bartolomei M.S. 2004. Transgenic RNAi reveals essential function for CTCF in $H 19$ gene imprinting. Science 303: 238.

Filippova G.N., Fagerlie S., Klenova E.M., Myers C., Dehner Y., Goodwin G., Neiman P.E., Collins S.J., and Lobanenkov V.V. 1996. An exceptionally conserved transcriptional repressor, CTCF, employs different combinations of zinc fingers to bind diverged promoter sequences of avian and mammalian c-myc oncogenes. Mol. Cell. Biol. 16: 2802.

Fitzpatrick G.V., Soloway P.D., and Higgins M.J. 2002. Regional loss of imprinting and growth deficiency in mice with a targeted deletion of KvDMR1. Nat. Genet. 32: 426.

Hajkova P., Erhardt S., Lane N., Haaf T., El-Maarri O., Reik W., Walter J., and Surani M.A. 2002. Epigenetic reprogramming in mouse primordial germ cells. Mech. Dev. 117: 15.

Hark A.T., Schoenherr C.J., Katz D.J., Ingram R.S., Levorse J.M., and Tilghman S.M. 2000. CTCF mediates methylationsensitive enhancer-blocking activity at the H19/Igf2 locus. Nature 405: 486.

Hata K., Okano M., Lei H., and Li E. 2002. Dnmt3L cooperates with the Dnmt3 family of de novo DNA methyltransferases to establish maternal imprints in mice. Development 129: 1983.

Howell C.Y., Bestor T.H., Ding F., Latham K.E., Mertineit C., Trasler J.M., and Chaillet J.R. 2001. Genomic imprinting disrupted by a maternal effect mutation in the Dnmt1 gene. Cell 104: 829.

Kanduri C., Fitzpatrick G., Mukhopadhyay R., Kanduri M., Lobanenkov V., Higgins M., and Ohlsson R. 2002. A differentially methylated imprinting control region within the Kcnq1 locus harbors a methylation-sensitive chromatin insulator. J. Biol. Chem. 277: 18106.

Kanduri C., Pant V., Loukinov D., Pugacheva E., Qi C.F., Wolffe A., Ohlsson R., and Lobanenkov V.V. 2000. Functional association of CTCF with the insulator upstream of the H19 gene is parent of origin-specific and methylation-sensitive. Curr. Biol. 10: 853.

Kaneda M., Okano M., Hata K., Sado T., Tsujimoto N., Li E., and Sasaki H. 2004. Essential role for de novo DNA methyltransferase $3 \mathrm{a}$ in paternal and maternal imprinting. Nature 429: 900.

Lalande M. 1996. Parental imprinting and human disease. Annu. Rev. Genet. 30: 173. 
La Salle S., Mertineit C., Taketo T., Moens P.B., Bestor T.H., and Trasler J.M. 2004. Windows for sex-specific methylation marked by DNA methyltransferase expression profiles in mouse germ cells. Dev. Biol. 268: 403.

Lee J., Inoue K., Ono R., Ogonuki N., Kohda T., Kaneko-Ishino T., Ogura A., and Ishino F. 2002. Erasing genomic imprinting memory in mouse clone embryos produced from day 11.5 primordial germ cells. Development 129: 1807.

Li E., Beard C., and Jaenisch R. 1993. Role for DNA methylation in genomic imprinting. Nature 366: 362.

Li L.L., Szeto I.Y., Cattanach B.M., Ishino F., and Surani M.A. 2000. Organization and parent-of-origin-specific methylation of imprinted Peg3 gene on mouse proximal chromosome 7. Genomics 63: 333.

Lin S.P., Youngson N., Takada S., Seitz H., Reik W., Paulsen M., Cavaille J., and Ferguson-Smith A.C. 2003. Asymmetric regulation of imprinting on the maternal and paternal chromosomes at the Dlk1-Gt12 imprinted cluster on mouse chromosome 12. Nat. Genet. 35: 97.

Loukinov D.I., Pugacheva E., Vatolin S., Pack S.D., Moon H., Chernukhin I., Mannan P., Larsson E., Kanduri C., Vostrov A.A., Cui H., Niemitz E.L., Rasko J.E., Docquier F.M., Kistler M., Breen J.J., Zhuang Z., Quitschke W.W., Renkawitz R., Klenova E.M., Feinberg A.P., Ohlsson R., Morse H.C., III, and Lobanenkov V.V. 2002. BORIS, a novel male germ-line-specific protein associated with epigenetic reprogramming events, shares the same 11-zinc-finger domain with $\mathrm{CTCF}$, the insulator protein involved in reading imprinting marks in the soma. Proc. Natl. Acad. Sci. 99: 6806.

Lucifero D., Mann M.R., Bartolomei M.S., and Trasler J.M. 2004. Gene-specific timing and epigenetic memory in oocyte imprinting. Hum. Mol. Genet. 13: 839.

Lucifero D., Mertineit C., Clarke H.J., Bestor T.H., and Trasler J.M. 2002. Methylation dynamics of imprinted genes in mouse germ cells. Genomics 79: 530.

Macleod D., Charlton J., Mullins J., and Bird A.P. 1994. Sp1 sites in the mouse aprt gene promoter are required to prevent methylation of the CpG island. Genes Dev. 8: 2282.

Mayer W., Niveleau A., Walter J., Fundele R., and Haaf T. 2000. Demethylation of the zygotic paternal genome. Nature 403: 501.

McGrath J. and Solter D. 1984. Completion of mouse embryogenesis requires both the maternal and paternal genomes. Cell 37: 179 .

McLaren A. 2003. Primordial germ cells in the mouse. Dev. Biol. 262: 1

Monk M., Boubelik M., and Lehnert S. 1987. Temporal and regional changes in DNA methylation in the embryonic, extraembryonic and germ cell lineages during mouse embryo development. Development 99: 371

Nicholls R.D. and Knepper J.L. 2001. Genome organization, function, and imprinting in Prader-Willi and Angelman syndromes. Annu. Rev. Genomics Hum. Genet. 2: 153.

Nicholls R.D., Ohta T., and Gray T.A. 1999. Genetic abnormalities in Prader-Willi syndrome and lessons from mouse models. Acta Paediatr. Suppl. 88: 99.

Obata Y. and Kono T. 2002. Maternal primary imprinting is established at a specific time for each gene throughout oocyte growth. J. Biol. Chem. 277: 5285.

Okano M., Bell D.W., Haber D.A., and Li E. 1999. DNA methyltransferases Dnmt3a and Dnmt3b are essential for de novo methylation and mammalian development. Cell 99: 247.

Oswald J., Engemann S., Lane N., Mayer W., Olek A., Fundele R., Dean W., Reik W., and Walter J. 2000. Active demethylation of the paternal genome in the mouse zygote. Curr. Biol. 10: 475.

Pant V., Mariano P., Kanduri C., Mattsson A., Lobanenkov V., Heuchel R., and Ohlsson R. 2003. The nucleotides responsible for the direct physical contact between the chromatin insulator protein CTCF and the H19 imprinting control region manifest parent of origin-specific long-distance insulation and methylation-free domains. Genes Dev. 17: 586.
Pfeifer K. 2000. Mechanisms of genomic imprinting. Am. J. Hum. Genet. 67: 777.

Plass C., Shibata H., Kalcheva I., Mullins L., Kotelevtseva N., Mullins J., Kato R., Sasaki H., Hirotsune S., Okazaki Y., Held W.A., Hayashizaki Y., and Chapman V. 1996. Identification of Grfl on mouse chromosome 9 as an imprinted gene by RLGS-M. Nat. Genet. 14: 106.

Rand E., Ben-Porath I., Keshet I., and Cedar H. 2004. CTCF elements direct allele-specific undermethylation at the imprinted H19 locus. Curr. Biol. 14: 1007.

Reik W. and Maher E.R. 1997. Imprinting in clusters: Lessons from Beckwith-Wiedemann syndrome. Trends Genet. 13: 330.

Rougier N., Bourc'his D., Gomes D.M., Niveleau A., Plachot M., Páldi A., and Viegas-Péquignot E. 1998. Chromosome methylation patterns during mammalian preimplantation development. Genes Dev. 12: 2108.

Santos F., Hendrich B., Reik W., and Dean W. 2002. Dynamic reprogramming of DNA methylation in the early mouse embryo. Dev. Biol. 241: 172 .

Schoenherr C.J., Levorse J.M., and Tilghman S.M. 2003. CTCF maintains differential methylation at the Igf2/H19 locus. Nat. Genet. 33: 66.

Shemer R., Birger Y., Riggs A.D., and Razin A. 1997. Structure of the imprinted mouse Snrpn gene and establishment of its parental-specific methylation pattern. Proc. Natl. Acad. Sci. 94: 10267.

Srivastava M., Hsieh S., Grinberg A., Williams-Simons L., Huang S.-P., and Pfeifer K. 2000. H19 and Igf2 monoallelic expression is regulated in two distinct ways by a shared cis acting regulatory region upstream of H19. Genes Dev. 14: 1186.

Stein P., Svoboda P., and Schultz R.M. 2003. Transgenic RNAi in mouse oocytes: A simple and fast approach to study gene function. Dev. Biol. 256: 187.

Stöger R., Kubicka P., Liu C.-G., Kafri T., Razin A., Cedar H., and Barlow D.P. 1993. Maternal-specific methylation of the imprinted mouse $\operatorname{Ig} f 2 r$ locus identifies the expressed locus as carrying the imprinting signal. Cell 73: 61.

Szabo P.E., Tang S.-H., Rentsendorj A., Pfeifer G.P., and Mann J.R. 2000. Maternal-specific footprints at putative CTCF sites in the $H 19$ imprinting control region give evidence for insulator function. Curr. Biol. 10: 607.

Szabo P.E., Tang S.H., Silva F.J., Tsark W.M., and Mann J.R. 2004. Role of CTCF binding sites in the Igf2/H19 imprinting control region. Mol. Cell. Biol. 24: 4791.

Thorvaldsen J.L. and Bartolomei M.S. 2000. Mothers setting boundaries. Science 288: 2145.

Thorvaldsen J.L., Duran K.L., and Bartolomei M.S. 1998. Deletion of the H19 differentially methylated domain results in loss of imprinted expression of H19 and Igf2. Genes Dev. 12: 3693.

Tremblay K.D., Duran K.L., and Bartolomei M.S. 1997. A 5' 2kilobase-pair region of the imprinted mouse H19 gene exhibits exclusive paternal methylation throughout development. Mol. Cell. Biol. 17: 4322.

Tycko B. 1999. Genomic imprinting and cancer. Results Probl. Cell Differ. 25: 133.

Ueda T., Abe K., Miura A., Yuzuriha M., Zubair M., Noguchi M., Niwa K., Kawase Y., Kona T., Matsuda Y., Fujimoto H., Shibata H., Hayashizaki Y., and Sasaki H. 2000. The paternal methylation imprint of the mouse $H 19$ locus is acquired in the gonocyte stage during foetal testis development. Genes Cells 5: 649 .

Verona R.I., Mann M.R., and Bartolomei M.S. 2003. Genomic imprinting: Intricacies of epigenetic regulation in clusters. Annu. Rev. Cell Dev. Biol. 19: 237.

Walsh C.P., Chaillet J.R., and Bestor T.H. 1998. Transcription of IAP endogenous retroviruses is constrained by cytosine methylation. Nat. Genet. 20: 116.

Yoon B.J., Herman H., Sikora A., Smith L.T., Plass C., and Soloway P.D. 2002. Regulation of DNA methylation of Rasgrf1. Nat. Genet. 30: 92. 


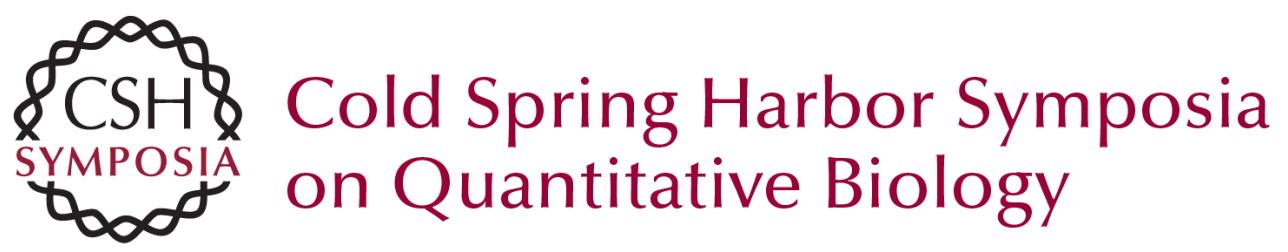

\title{
Genomic Imprinting: Antagonistic Mechanisms in the Germ Line and Early Embryo
}

\author{
A.M. FEDORIW, N.I. ENGEL and M.S. BARTOLOMEI \\ Cold Spring Harb Symp Quant Biol 2004 69: 39-46 \\ Access the most recent version at doi:10.1101/sqb.2004.69.39
}

References This article cites 64 articles, 17 of which can be accessed free at: http://symposium.cshlp.org/content/69/39.full.htmI\#ref-list-1

\section{License}

Email Alerting Receive free email alerts when new articles cite this article - sign up in Service the box at the top right corner of the article or click here. 